
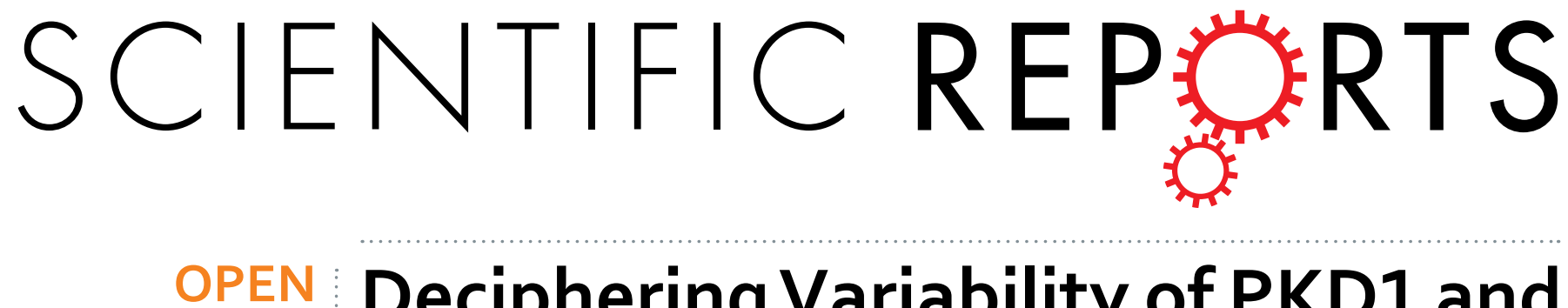

\title{
Deciphering Variability of PKD1 and PKD2 in an Italian Cohort of 643 Patients with Autosomal Dominant \\ Polycystic Kidney Disease (ADPKD)
}

Received: 02 March 2016

Accepted: 08 July 2016

Published: 08 August 2016
Paola Carrera ${ }^{1,2}$, Silvia Calzavara ${ }^{2}$, Riccardo Magistroni ${ }^{3,4}$, Johan T. den Dunnen ${ }^{5}$, Francesca Rigo ${ }^{1}$, Stefania Stenirri ${ }^{1}$, Francesca Testa ${ }^{4}$, Piergiorgio Messa ${ }^{6}$, Roberta Cerutti ${ }^{6}$, Francesco Scolari ${ }^{7}$, Claudia Izzi ${ }^{7}$, Alberto Edefonti ${ }^{8}$, Susanna Negrisolo ${ }^{9}$, Elisa Benetti ${ }^{10}$, Maria Teresa Sciarrone Alibrandi ${ }^{11}$, Paolo Manunta ${ }^{11}$, Alessandra Boletta ${ }^{3}$ \& Maurizio Ferrari ${ }^{1,2,12}$

Autosomal Dominant Polycystic Kidney Disease (ADPKD) is the most common hereditary kidney disease. We analysed $P K D 1$ and $P K D 2$, in a large cohort of 440 unrelated Italian patients with ADPKD and 203 relatives by direct sequencing and MLPA. Molecular and detailed phenotypic data have been collected and submitted to the PKD1/PKD2 LOVD database. This is the first large retrospective study in Italian patients, describing 701 variants, 249 (35.5\%) already associated with ADPKD and 452 (64.5\%) novel. According to the criteria adopted, the overall detection rate was $80 \%(352 / 440)$. Novel variants with uncertain significance were found in $14 \%$ of patients. Among patients with pathogenic variants, in $301(85.5 \%)$ the disease is associated with PKD1, $196(55.7 \%)$ truncating, 81 (23\%) non truncating, 24 (6.8\%) IF indels, and in 51 (14.5\%) with PKD2. Our results outline the high allelic heterogeneity of variants, complicated by the presence of variants of uncertain significance as well as of multiple variants in the same subject. Classification of novel variants may be particularly cumbersome having an important impact on the genetic counselling. Our study confirms the importance to improve the assessment of variant pathogenicity for ADPKD; to this point databasing of both clinical and molecular data is crucial.

Autosomal Dominant Polycystic Kidney Disease (ADPKD) is the most common genetic nephro-pathology in humans, with a prevalence of $1 / 400-1000$ subjects at birth $^{1}$, and characterized by bilateral renal cyst formation. Cysts develop by massive enlargement of every segment of the nephron resulting in renal failure leading to dialysis or transplant ${ }^{1}$. The observed phenotypic variability in ADPKD involves differences in the rate of loss of glomerular filtration, the age of reaching end-stage renal disease (ESRD), the occurrence of hypertension, symptomatic extra-renal cysts, and subarachnoid hemorrhage from intracranial 'berry' aneurysm.

The majority of ADPKD patients carry a germ-line variant in the PKD1 gene (ADPKD1- MIM173900) on chromosome 16p13, and 15 to $20 \%$ of patients harbor a PKD2 variant on chromosome 4q21 (ADPKD2-MIM613095).

${ }^{1}$ IRCCS San Raffaele Scientific Institute, Division of Genetics and Cell Biology, Unit of Genomics for Human Disease Diagnosis, Milan, Italy. ${ }^{2}$ Laboratory of Clinical Molecular Biology, Ospedale San Raffaele, Milan, Italy. ${ }^{3}$ IRCCS San Raffaele Scientific Institute, Division of Genetics and Cell biology, Molecular Basis of Polycystic Kidney Disease Unit, Milan, Italy. ${ }^{4}$ Division of Nephrology and Dialysis A.O. U. Policlinico, University of Modena and Reggio Emilia, Modena, Italy. ${ }^{5}$ Depts. Clinical Genetics and Human Genetics, Leiden University Medical Centre, Netherlands. ${ }^{6}$ Dept. of Nephrology, Urology and Transplant, IRCCS Cà Granda Policlinico, Milan, Italy. ${ }^{7}$ Center for Prenatal Diagnosis and Nephrology, A.O. Spedali Civili, Brescia, Italy. ${ }^{8}$ Dept. of Paediatric Nephrology and Dialysis, IRCCS Cà Granda Policlinico, Milan, Italy. ${ }^{9}$ Laboratory of Immunopathology and Molecular Biology of the Kidney, Dept. SDB, Padova, Italy. ${ }^{10}$ Pediatric Nephrology, Dialysis and Transplant Unit; Department of Women's and Children's Health, Padova, Italy. ${ }^{11}$ Vita-Salute San Raffaele University, chair of Nephrology, IRCCS Ran Raffaele Scientific Institute, Genomics of Renal Disease and Hypertension Unit, Milan, Italy. ${ }^{12}$ Vita-Salute San Raffaele University, chair of Clinical Pathology, Milan, Italy. Correspondence and requests for materials should be addressed to P.C. (email: carrera.paola@hsr.it) 
Disease manifestations are characterized by a high inter and intra familial variability, raising the possibility of the existence of modifiers for the disease ${ }^{1}$. Clinical symptoms usually do not arise until middle age. Recently, in a minor subset of patients, in whom ADPKD manifests very early in life and presents as a much more severe disorder ${ }^{2}$ it was demonstrated that early and severe ADPKD is not strictly confined to individuals with a PKD1 variant as previously thought but can also occur in other genetic combinations: patients with a $P K D 2$ variant $^{3}$, coinheritance in trans of an incompletely penetrant $P K D 1$ allele with an inactivating $P K D 1$ variant $^{4}$. In addition, among those cases with early-onset ADPKD, are cases with clinical features sometimes indistinguishable from those with a typical presentation of autosomal recessive polycystic kidney disease (ARPKD) and variants in the PKHD1 gene ${ }^{5}$.

Until recent, the observation that ADPKD1 causes more severe disease with an average age of 54 years at ESRD (versus 74 years for ADPKD2) was the only correlation between genotype and phenotype ${ }^{1}$. In a more recent paper, authors found a correlation between the type of $P K D 1$ variant and renal survival. The median age at onset of ESRD was 55 years for carriers of a truncating variant and 67 years for carriers of a non-truncating variant ${ }^{6}$

Taken all together, these studies highlight the potential high complexity of the genetics of ADPKD and warrant the need for studies aimed at characterizing the different variants identified. The high level of allelic heterogeneity, the various types of variants observed in PKD1 and PKD2 rise issues with respect to their classification in particular for rare and novel non-truncating variants. Increasing the knowledge on the spectrum of variants and sharing of data would definitely be helpful to improve our capability for variant classification, correlation to clinical features and stratification of patients.

Molecular analysis of ADPKD has proved difficult for a long time, because of genetic and allelic heterogeneity, for the presence of GC-rich regions and more importantly because the $5^{\prime}$ region of $P K D 1$, from exons 1 to 33 , is duplicated in six highly homologous pseudogenes, all located in chromosome $16 \mathrm{p}^{7,8}$. To date, the wider variant scanning diagnostic studies of the entire $P K D 1$ and $P K D 2$ genes, has been performed by DHPLC and/or sequencing, in combination with other methods for the identification of large rearrangements ${ }^{8,9}$. To date, the four larger studies reported the distribution of PKD1 and PKD2 variants in 202 (CRISP, USA) ${ }^{10}, 700$ (GENKYST, France) ${ }^{9}$, 220 (TGESP, Canada) ${ }^{11}$ and 1119 patients (HALT-PKD and CRSP, USA) ${ }^{12}$.

Aim of the present work was to realize a comprehensive description of all the genetic variation of PKD1 and $P K D 2$ in a large cohort of Italian patients affected by ADPKD: 643 subjects were analysed by a semi-automated Sanger direct sequencing protocol, followed by multiple ligation probe assay (MLPA) analysis. A second aim was to establish a database for ADPKD following existing standards, linked with international resources and suited for the collection of patient-specific variants and key clinical features.

\section{Results and Discussion}

In the present study, a semi-automated direct sequencing method was applied for detection of variants in PKD1 and $P K D 2$ genes in a cohort of Italian patients affected by ADPKD. One of the major difficulties of $P K D 1$ sequencing is the specific selection of the functional gene due to the presence of six highly homologous pseudogenes. In the present study, we applied protocols previously described by Phakdeekitcharoen B. and coauthors ${ }^{7}$ for the specific amplification of PKD1 by Long-Range PCR amplification, assuming they are PKD1-specific. Nevertheless, to avoid amplification of the PKD1 pseudogenes, the design of primers, either for the Long-Range or for nested PCR, was verified on a multiple alignment (Lasergene) between PKD1 and the 6 pseudogenes. In order to select primers complementary solely to PKD1, primers were located in regions where a sequence divergence with the pseudogenes is present, with the differences located at the most $3^{\prime}$ nucleotidic position. As an example, we added the Supplementary Figure S1, where the sequencing result of the specific amplification of PKD1 exon 10 with our primers is shown and compared with the result of an unspecific amplification.

By looking at sequencing results, we first considered known variants; we grouped either common or rare variants previously classified as likely neutral as benign variants. A total of $113 P K D 1$ and 8 PKD2 variants were identified, with an average of 11 per patient in PKD1 and 2 per patient in PKD2. In Supplementary Table S1, benign variants observed in PKD1 are listed with the minor allele frequency (MAF) in our ADPKD cohort, in the 1000 genomes (general and TSI Italian sub-population) and the PKDB classification. By comparing the group of patients and the general populations, none of the variants showed marked differences in their frequencies. We only noticed that sometimes the MAF observed in the group of the patients was more similar to the general MAF reported in 1000 genomes than to that in the TSI subgroup.

In addition to known benign variants, also rare and novel variants were identified; in particular, a total of 701 DNA novel and already described pathogenic variants have been detected: $625(89.2 \%)$ from PKD1 and 76 (10.8\%) from PKD2, confirming the marked allelic heterogeneity of these genes. In Table 1, the different classes of variations identified in familial and in sporadic subgroups are listed for each gene. Variants were present in $94.3 \%$ of probands (415/440).

Of the total 701 changes, 249 (35.5\%) were variants already described (http://pkdb.mayo.edu), 216 (30.8\%) in PKD1 and 33 (4.7\%) in PKD2 (Suppl. Table S2). Among these also variants classified as likely neutral. The remaining 452 (64.5\%) were new changes, 409 (58.4\%) in PKD1 and 43 (6.1\%) in PKD2. Table 2, summarizes the novel variants identified in patients, distributed in classes.

For all the identified variants a classification with respect to pathogenicity was attempted, as described in the methods section. Variants have been classified as definite pathogenic (Supp. Table S3), highly likely pathogenic (Supp. Table S4), likely pathogenic (Suppl. Table S5), of uncertain significance (Suppl. Table S6), or likely non-pathogenic (Suppl. Table S7).

By combining results from known pathogenic variants, current classification and family studies we were able to detect the pathogenic variant in 352 patients. 


\begin{tabular}{|l|c|c|c|c|}
\hline \multirow{2}{*}{ Cases } & \multicolumn{2}{|c|}{ PKD1 } & \multicolumn{2}{c|}{ PKD2 } \\
\cline { 2 - 5 } & Familial & Sporadic & Familial & Sporadic \\
\hline Large rearrangements & 2 & 1 & 0 & 1 \\
\hline Canonic splicing & 17 & 7 & 8 & 2 \\
\hline Frameshift & 73 & 19 & 10 & 0 \\
\hline Nonsense & 60 & 17 & 15 & 3 \\
\hline In frame indels $\geq 5$ & 4 & 0 & 0 & 0 \\
\hline In frame indels $<5$ & 13 & 8 & 1 & 0 \\
\hline Missense & 165 & 51 & 12 & 6 \\
\hline Intronic & 87 & 24 & 10 & 3 \\
\hline Synonym & 54 & 23 & 3 & 2 \\
\hline Total & 475 & 150 & 59 & 17 \\
\hline Total F+S & \multicolumn{2}{|c|}{625} & & 76 \\
\hline
\end{tabular}

Table 1. Total number of PKD1 and PKD2 variants identified, grouped in classes.

\begin{tabular}{|l|c|c|c|c|}
\hline \multirow{2}{*}{ Cases } & \multicolumn{2}{|c|}{ PKD1 } & \multicolumn{2}{c|}{ PKD2 } \\
\cline { 2 - 5 } & Familial & Sporadic & Familial & Sporadic \\
\hline Large rearrangements & 0 & 1 & 0 & 0 \\
\hline Canonic splice sites & 8 & 5 & 3 & 1 \\
\hline Frameshift & 46 & 12 & 5 & 0 \\
\hline Nonsense & 26 & 9 & 5 & 2 \\
\hline In frame indels $\geq 5$ & 3 & 0 & 0 & 0 \\
\hline In frame indels $<5$ & 6 & 2 & 0 & 0 \\
\hline Missense & 104 & 36 & 6 & 5 \\
\hline Intronic & 67 & 19 & 10 & 3 \\
\hline Synoyim & 46 & 19 & 2 & 1 \\
\hline Total & 306 & 103 & 31 & 12 \\
\hline Total F+S & & 409 & & 43 \\
\hline
\end{tabular}

Table 2. PKD1 and PKD2 novel variants identified, grouped in classes.

In Table 3 the types and frequencies of definite and probable pathogenic variants are reported. In PKD1, frameshifting variants were the more represented, accounting for $30 \%$, while in PKD2 the nonsense was the major type with a 35\% frequency. By comparing definite pathogenic variants, they were more frequently detected in PKD2 (76\%) than in PKD1 (66\%). In Table 3, data on PKD1 and PKD2 from the previous large CRISP ${ }^{10}$. GENKYST $^{9}$. TGESP ${ }^{11}$ and HALT-PKD and CRISP ${ }^{12}$ studies, on 180, 442, 188 and 1034 pathogenic variants, respectively, are reported and compared to the present study. In $P K D 1$, truncating variants (grouping frameshift, nonsense, canonic splice site changes and large rearrangements) were the more represented in all the studies, with the TGESP ${ }^{11}$ study displaying the lower percentage (38.3\%). On the contrary the GENKYST ${ }^{12}$ study showed the lower frequency of non-truncating variants. In PKD2 the results were similar with the exception of the TGESP study showing a frequency of variants 2 times higher compared to the other cohorts.

In the present study the overall detection rate was $80 \%(352 / 440)$, with $83 \%(266 / 320)$ in the group of patients with familiarity, and $72 \%(86 / 120)$ in the group of sporadic patients (Table 3$)$.

Among patients with pathogenic variants, 301 (85.5\%) carry a PKD1 variant, and $51(14.5 \%)$ carry a PKD2 variant, in agreement to previous reports. Moreover, in previous studies, the overall detection rate was higher: $89.1 \%$ in the CRISP study ${ }^{10} .89 .9 \%$ in the GENKYST study ${ }^{9}, 84.5 \%$ in the TGESP ${ }^{11}$ study, and $92.4 \%$ in the HALT-PKD and CRISP ${ }^{12}$ study. In our cohort, pathogenic variants were not found in $20 \%(\mathrm{n}=88)$ of the 440 unrelated patients. Among these, in 25 patients (6\%), only known benign variants were present. In the remaining 63 patients (14\%), novel variants with uncertain significance were found, mainly missense variants. To explain this lower detection rate, it is possible to argue that the classification system adopted was imperfect, or that our population may be enriched by other classes of pathogenic variants (such as variants located in regulatory regions), not detected. Another explanation may be that in our population the collection of samples was somehow biased. Actually, our cohort was enriched in subjects with an uncertain clinical diagnosis $(96 / 440,21.8 \%)$, since one of the criteria to access genetic testing was the clinical atypical presentation. In this subgroup we obtained a lower detection rate $(62 / 96,64.6 \%)$ of definitely pathogenic variants, a lower proportion of patients with variants with uncertain significance $(11,11.4 \%)$ and a higher proportion of patients without variants $(23,24 \%)$ who may have other non-ADPKD cystic kidney disease explaining their phenotype.

In order to check the sensitivity and specificity of our genetic testing in relation to the clinical phenotypes, we performed a correlation between the pathogenic variants and the clinical characteristics of patients, when available. Considering the clinical diagnosis and the reason for testing, as reported in Supplementary Table S8, the correlation was done for patients with positive diagnostic criteria in the presence (true positives) or in the 


\begin{tabular}{|c|c|c|c|c|c|}
\hline \multicolumn{2}{|l|}{ Type of variant } & \multicolumn{2}{|c|}{ PKD1 } & \multicolumn{2}{|c|}{ PKD2 } \\
\hline \multicolumn{6}{|l|}{ Definite pathogenic } \\
\hline \multicolumn{2}{|c|}{ Large rearrangements } & 3 & $1 \%$ & 1 & $2 \%$ \\
\hline \multicolumn{2}{|l|}{ Frameshift } & 92 & $30 \%$ & 10 & $19.5 \%$ \\
\hline \multicolumn{2}{|l|}{ Nonsense } & 77 & $26 \%$ & 18 & $35 \%$ \\
\hline \multicolumn{2}{|l|}{ Canonic splicing } & 24 & $8 \%$ & 10 & $19.5 \%$ \\
\hline \multicolumn{2}{|c|}{ In-frame $\geq 5$ aminoacids } & 4 & $1 \%$ & 0 & \\
\hline \multicolumn{6}{|c|}{ Probable pathogenic $(\mathrm{HLP}+\mathrm{LP})$} \\
\hline \multicolumn{2}{|l|}{ Missense } & 75 & $25 \%$ & 9 & $18 \%$ \\
\hline \multicolumn{2}{|c|}{ In-frame $<5$ aminoacids } & 20 & $7 \%$ & 1 & $2 \%$ \\
\hline \multicolumn{2}{|l|}{ Atypical splicing } & 6 & $2 \%$ & 2 & $4 \%$ \\
\hline \multicolumn{6}{|c|}{ Pathogenic variants spectra, comparison with previous large studies } \\
\hline \multirow{2}{*}{\multicolumn{6}{|c|}{\begin{tabular}{|l|} 
\\
PKD1
\end{tabular}}} \\
\hline & & & & & \\
\hline \multicolumn{6}{|l|}{ truncating } \\
\hline nontruncating & $81(23 \%)$ & $41(22.8 \%)$ & $73(16.5 \%)$ & $51(27.1 \%)$ & $223(21.6 \%)$ \\
\hline IF indel & $24(6.8 \%)$ & $9(5 \%)$ & $29(6.6 \%)$ & $8(4.3 \%)$ & $54(5.2 \%)$ \\
\hline PKD2 & $51(14.5 \%)$ & $27(15 \%)$ & $54(12.2 \%)$ & $57(30.3 \%)$ & $165(15.9 \%)$ \\
\hline \multicolumn{6}{|c|}{ Classification of PKD1 and PKD2 variants and detection rates } \\
\hline & \multicolumn{2}{|c|}{ PKD1 } & \multicolumn{2}{|c|}{ PKD2 } & \multirow{2}{*}{$P K D 1+P K D 2$} \\
\hline & DP & HLP + LP & DP & HLP + LP & \\
\hline Familial $(\mathrm{n}=320)$ & $154(48 \%)$ & $73(23 \%)$ & $33(10 \%)$ & $6(2 \%)$ & $266(83 \%)$ \\
\hline Sporadic $(\mathrm{n}=120)$ & $46(38 \%)$ & $28(24 \%)$ & $6(5 \%)$ & $6(5 \%)$ & $86(72 \%)$ \\
\hline Total DP & 200 & & 39 & & $239(54 \%)$ \\
\hline \multirow[t]{2}{*}{ Total HLP + LP } & & 101 & & 12 & $113(26 \%)$ \\
\hline & \multicolumn{2}{|c|}{ Total PKD1 $301(85.5 \%)$} & \multicolumn{2}{|c|}{ Total PKD2 51 (14.5\%) } & $352(80 \%)$ \\
\hline
\end{tabular}

Table 3. Distribution of definite and probable pathogenic variants in 352 probands. ${ }^{\circ} \mathrm{All}$ in-frame indels grouped together. ${ }^{*}$ Total probands/families with pathogenic variants. ${ }^{\S}$ Total patients with pathogenic variants truncating: frameshift, nonsense, canonic splice site, large rearrangements nontruncating: missense, atypical splicing. DP: definite pathogenic; HLP: highly likely pathogenic; LP: likely pathogenic.

absence (false negatives) of a pathogenic variant and for patients not at risk and negative diagnostic criteria in the presence (false positive) or in the absence (true negatives) of a pathogenic variant. We reached $85 \%$ sensitivity and $100 \%$ specificity.

In order to evaluate the performance of the prediction for missense variants adopted in the present study, a comparison with the results of the prediction reported in PKDB was done. Classification in this study differed from that in the PKDB for 14 out of 83 previously reported missense variants, showing a $83 \%$ concordance. As reported in Table 4, among the 14 discrepant variants, 9 were classified as uncertain with our prediction model and likely or highly likely pathogenic in PKDB, indicating a higher grade of uncertainty with our prediction. In 4 cases, variants classified as indeterminate in PKDB resulted as likely pathogenic in this study. Noticeably for three of these our result was only slightly different since a border-line scoring $(=14)$ was obtained. In only 1 case (c.10678G $>$ A) the prediction was completely opposite: likely pathogenic in this study and likely neutral in PKDB; by looking at concurrent variants in the patient it is possible to observe that in this case a truncating variant was present, thus reinforcing probability for the neutral nature of the c.10678G $>A$ variant. Taking into account previous and present classification, a prudent conclusion would be that prediction of missense variants is still imperfect and that a consensus on the criteria for classification of pathogenicity would be useful.

Among the 352 different pathogenic variants in PKD1 and PKD2, 65 (18.5\%) of them were found in at least 2 unrelated patients. Table 5 lists the variants that were found in at least 3 unrelated patients, 10 in PKD1 and 4 in PKD2. Of the 14 variants, 8 are single nucleotide substitutions, 5 are small deletions, 1 is a single nucleotide duplication. Only three of them were previously described as frequent/recurrent variants in PKD1: c.2180T $>$ C; c.5014_5015delAG; and c.8311G $>\mathrm{A}^{9,10}$. The three novel pathogenic variants c.3607C $>\mathrm{T}$ and c.11354G $>\mathrm{C}$ in $P K D 1$ and c.637C $>$ T in $P K D 2$ are very interesting since they may represent Italian clusters but, unfortunately, we were not able to define if the origin of these variants was from a common ancestor.

In some cases, the family study helped us to support the pathogenic role of a variant. This was the case for the c. 1202-9G > A intronic variant (Fig. 1) and for the synonymous c. 2097G > T variant (Fig. 2), in PKD1.

In line with allelic heterogeneity, in many patients more than one candidate variant was observed (Table 6). In the majority of cases, it was not possible to perform segregation analysis within the family, thus the most likely candidate pathogenic variant was inferred based on its nature (truncating, not truncating) and based on predictions, as illustrated in the Methods. Nevertheless, in few cases, more than one variant classified as pathogenic, definitely or likely, novel or described, was present in the same subject, with both of them in PKD1 or one in PKD1 and the second in PKD2 (Table 7). In these cases, various combinations of variants have been found: coexistence of two definitely pathogenic variants or of one definitely pathogenic with a likely pathogenic variant. 


\begin{tabular}{|c|c|c|c|c|c|c|c|c|c|c|c|c|}
\hline \multicolumn{13}{|l|}{ PKD1 } \\
\hline $\begin{array}{l}\text { DNA change } \\
\text { (cDNA) }\end{array}$ & protein change & $\begin{array}{l}\text { mutation } \\
\text { type }\end{array}$ & $\begin{array}{l}\text { exon/ } \\
\text { IVS }\end{array}$ & Gs & AGVGDs & PP2s & SIFTs & MTs & $\begin{array}{l}\text { total } \\
\text { score }\end{array}$ & $\begin{array}{c}\text { concurrent variants/ } \\
\text { classification }\end{array}$ & $\begin{array}{l}\text { prediction in this } \\
\text { study }\end{array}$ & \begin{tabular}{|l|}
$\begin{array}{l}\text { PKDB } \\
\text { classification }\end{array}$ \\
\end{tabular} \\
\hline c. $224 \mathrm{C}>\mathrm{T}$ & p.Ser75Phe & missense & 2 & 155 & $\mathrm{C} 25$ & B & $\mathrm{D}$ & $\mathrm{D}$ & 12 & no & uncertain significance & $\begin{array}{l}\text { highly likely } \\
\text { pathogenic }\end{array}$ \\
\hline c. $827 \mathrm{C}>\mathrm{T}$ & p.Thr276Ile & missense & 5 & 89 & $\mathrm{C} 0$ & B & $\mathrm{T}$ & $\mathrm{T}$ & 2 & $\begin{array}{l}\text { p.Glu2771Lys/HLP } \\
\text { p.Leu726Phe/US } \\
\text { p.Asp1332Asn/US }\end{array}$ & uncertain significance & likely pathogenic \\
\hline c. $1606 \mathrm{G}>\mathrm{A}$ & p.Gly536Ser & missense & 7 & 50 & $\mathrm{C} 0$ & $\mathrm{D}$ & $\mathrm{T}$ & $\mathrm{D}$ & 8 & no & uncertain significance & likely pathogenic \\
\hline c. $5830 \mathrm{G}>\mathrm{A}$ & p.Gly1944Arg & missense & 15 & 125 & $\mathrm{C} 0$ & $\mathrm{D}$ & $\mathrm{D}$ & $\mathrm{D}$ & 14 & p.Ala3053Thr/LP & likely pathogenic & indeterminate \\
\hline c. $6062 \mathrm{~T}>\mathrm{C}$ & p.Leu2021Pro & missense & 15 & 98 & $\mathrm{C} 0$ & $\mathrm{D}$ & $\mathrm{D}$ & $\mathrm{D}$ & 14 & no & likely pathogenic & indeterminate \\
\hline c. $6151 \mathrm{C}>\mathrm{T}$ & p.Arg2051Cys & missense & 15 & 180 & $\mathrm{C} 15$ & B & $\mathrm{T}$ & $\mathrm{T}$ & 3 & $\begin{array}{c}\text { c.11537+2T }>\mathrm{A} / \\
\text { DP p.Arg3277Cys/ } \\
\text { Hypomorphic }\end{array}$ & uncertain significance & likely pathogenic \\
\hline c. $7271 \mathrm{C}>\mathrm{T}$ & p.Thr2424Met & missense & 18 & 81 & C65 & $\mathrm{D}$ & $\mathrm{D}$ & $\mathrm{D}$ & 20 & no & likely pathogenic & indeterminate \\
\hline c. $9047 \mathrm{~A}>\mathrm{G}$ & p.Gln3016Arg & missense & 25 & 43 & $\mathrm{C} 35$ & $\mathrm{D}$ & $\mathrm{T}$ & $\mathrm{D}$ & 11 & no & uncertain significance & $\begin{array}{l}\text { highly likely } \\
\text { pathogenic }\end{array}$ \\
\hline c. $9361 \mathrm{G}>\mathrm{A}$ & p.Glu3121Lys & missense & 26 & 56 & C55 & $\mathrm{D}$ & $\mathrm{T}$ & $\mathrm{D}$ & 13 & p.Arg4154Cys/HLP & uncertain significance & likely pathogenic \\
\hline c. $9412 \mathrm{G}>\mathrm{A}$ & p.Val3138Met & missense & 27 & 21 & $\mathrm{C} 15$ & $\mathrm{D}$ & $\mathrm{D}$ & $\mathrm{D}$ & 13 & no & uncertain significance & likely pathogenic \\
\hline c.10678G $>A$ & p.Gly3560Arg & missense & 36 & 125 & $\mathrm{C} 0$ & $\mathrm{D}$ & $\mathrm{T}$ & $\mathrm{D}$ & 14 & p.Phe3257Leufs*57/DP & likely pathogenic & likely neutral \\
\hline c. $10951 \mathrm{G}>\mathrm{A}$ & p.Gly3651Ser & missense & 37 & 56 & C55 & $\mathrm{D}$ & $\mathrm{T}$ & $\mathrm{D}$ & 13 & p.Arg2765His/US & uncertain significance & $\begin{array}{l}\text { highly likely } \\
\text { pathogenic }\end{array}$ \\
\hline c. $11258 \mathrm{G}>\mathrm{A}$ & p.Arg3753Gln & missense & 39 & 43 & $\mathrm{C} 0$ & $\mathrm{D}$ & $\mathrm{T}$ & $\mathrm{D}$ & 8 & p.Pro1429Ser/US & uncertain significance & $\begin{array}{l}\text { highly likely } \\
\text { pathogenic }\end{array}$ \\
\hline c. $12826 \mathrm{C}>\mathrm{T}$ & p.Arg4276Trp & missense & 46 & 101 & $\mathrm{C} 0$ & $\mathrm{D}$ & $\mathrm{D}$ & $\mathrm{D}$ & 14 & p.Leu56Argfs*15/DP & likely pathogenic & indeterminate \\
\hline
\end{tabular}

Table 4. Variants with a discrepant classification. DP: definitely pathogenetic; HLP: highly likely pathogenic; LP: likely pathogenic; US: uncertain significance.

\begin{tabular}{|c|c|c|c|c|c|}
\hline cDNA & Protein & Exon/IVS & Number of unrelated patients & Prediction & Described/Novel \\
\hline \multicolumn{6}{|l|}{$P K D 1$} \\
\hline c.856_862delTCTGGCC & p.Gly $287 \mathrm{fs} * 1$ & 5 & 4 & definitely pathogenic & Described \\
\hline c. $2180 \mathrm{~T}>\mathrm{C}$ & p.Leu727Pro & 11 & 4 & likely pathogenic & Described \\
\hline c.5014_5015delAG & p.Arg1672Glyfs*97 & 15 & 9 & definitely pathogenic & Described \\
\hline c. $3607 \mathrm{C}>\mathrm{T}$ & p.Gln1203* & 15 & 3 & definitely pathogenic & Novel \\
\hline c. $8311 \mathrm{G}>\mathrm{A}$ & p.Glu2771Lys & 23 & 5 & highly likely pathogenic & Described \\
\hline c.8935_8937delTTC & p.Phe2979del & 24 & 3 & highly likely pathogenic & Described \\
\hline c.11134delC & p.Arg3712Glyfs*14 & 38 & 3 & definitely pathogenic & Described \\
\hline c.11354G $>C$ & p.Trp3785Ser & 40 & 3 & likely pathogenic & Novel \\
\hline c.6916-9G>A & & IVS15 & 3 & highly likely pathogenic & Described \\
\hline c.8017-2_-1delAG & & IVS21 & 3 & definitely pathogenic & Described \\
\hline \multicolumn{6}{|l|}{ PKD2 } \\
\hline c. $637 \mathrm{C}>\mathrm{T}$ & p.Arg213* & 2 & 6 & definitely pathogenic & Novel \\
\hline c. $916 \mathrm{C}>\mathrm{T}$ & p.Arg306* & 4 & 3 & definitely pathogenic & Described \\
\hline c.2159dupA & p.Asn720Lysfs*5 & 11 & 3 & definitely pathogenic & Described \\
\hline c. $1094+1 \mathrm{G}>\mathrm{A}$ & p.? & IVS4 & 3 & definitely pathogenic & Described \\
\hline
\end{tabular}

Table 5. Pathogenic variants found in at least 3 unrelated patients.

The simplest interpretation would be to declare as pathogenic only the variant with a definite classification; nevertheless coexistence of a definite variant with another candidate variant has to be taken into account since compound heterozygosity has been already observed in patients with early and severe ADPKD ${ }^{4,5}$. Thus, the results of molecular testing for ADPKD have to be considered very carefully and have important implications for genetic counselling since definition of risk in relatives may be cumbersome. In order to define the contribution of each variant to the phenotype, segregation analysis and correlation with the phenotype in the family should be always advised. An example of such a situation is showed in Fig. 3. In this family, segregation analysis showed that the p.Val2897delinsAlaAsnSer in-frame del/ins was inherited from the health father while the definite pathogenic frameshift variant p.Val4038Glyfs* 118 was transmitted from the affected mother. The hypothesis that the early onset in the proband may be influenced by the paternal variant, was taken into account and a pathogenic role of the p.Val2897delinsAlaAsnSer, classified as highly likely pathogenic with the criteria adopted ${ }^{9}$, was not excluded. Actually, an argument against its pathogenic role was that it is located near the variant p.2894insAlaAsnSer, previously classified as a polymorphic variant based on segregation studies and on evidence of a recent origin in the 


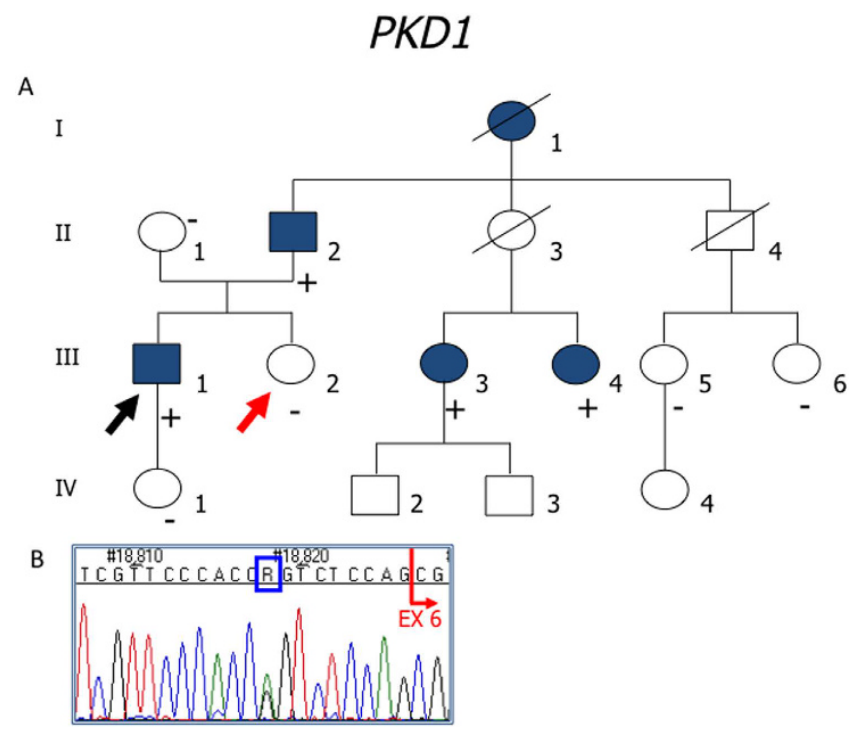

Figure 1. (A) In this family, the proband (III-1, black arrow) with ESRD, inherited from the father (II-2) and shared only with affected relatives (III-3, III-4) the intronic PKD1 variant: c.1202-9G $>$ A. None of the analyzed healthy subjects in the family (II-1, III-2, III-5, III-6, IV-1) had this variant; (B) Sequence chromathogram; abolishing of the nearby splice acceptor site was predicted in silico (www.fruitfly.org). Genetic testing was important to support classification of the intronic variant as highly likely pathogenic and to identify the III-2 subject (red arrow) as a potential donor for kidney transplantation in the patient. Unfortunately it was not possible to collect the proper samples for RT-PCR. Filled symbol: ADPKD; empty symbol: asymptomatic; $(+)$ sign indicates a subject heterozygous for the c.1202-9G $>$ A variant; $(-)$ sign indicates a subject without the variant.

human genome ${ }^{10}$. Thus, at the moment, classification of the p.Val2897delinsAlaAsnSer remains doubtful, and further evidences are needed in order to conclude in favour or against pathogenicity.

Search for large rearrangements by MLPA revealed one case with deletion of the whole PKD2, the fifth reported to date ${ }^{13,14,9}$, two cases with deletion of the whole $P K D 1$ and one case with a partial PKD1 deletion (c.216793_427del1431), described in Fig. 4. This finding is in line with previously reported frequencies $(1-3 \%)^{9,15}$. Nevertheless, we have to notice that commercial MLPA assays, like that used in the present study, do not include probes specific for all exonic regions, therefore we cannot exclude the occurrence of false negative results.

In Fig. 5 a family with evidence of germinal mosaicism is shown. Somatic mosaicism has been described in $40 \%$ of PKD1/TSC contiguous gene deletion syndrome patients, in association with large rearrangements involving the adjacent $P K D 1$ and $T S C$ genes ${ }^{14}$. In our family, the disease was associated with a truncating variant, the presence of two affected siblings suggested a mosaicism in the parents, likely confined to the germ-line and excluded the hypothesis of a de novo mutation, with an impact on their reproductive risk.

Considering the high allelic variability observed in patients, and taking into account the reported correlation between the various classes of pathogenic variants and the onset of ESRD ${ }^{6,11,12}$, we calculated the proportion of patients with or without ESRD in the subgroups with PKD1 truncating, not truncating, IF indel and PKD2. As shown in Table 8, the proportion of PKD1 truncating variants is higher in the group of patients with ESRD. By comparing the age at onset of ESRD in patients harbouring PKD1 or PKD2 pathogenic variants, we observed a significant difference between the subgroups ( $p=0.0194$ log rank) (Fig. 6), with an earlier ESRD onset in PKD1 patients. These results are in line with previous observations; nevertheless, in consideration of the retrospective nature of these clinical data, we feel that a more detailed and complete collection of clinical data will be necessary to assess the prognostic value in term of renal survival.

\section{Conclusions}

This is the first Italian study performed on a large collection of 440 probands with ADPKD, aimed at identifying the molecular variability in PKD1 and PKD2. The resulting genetic variants as well as key clinical data have been collected in a database established at the Leiden Open Variation Database ${ }^{16}$. These set data will be relevant for classification of variants as well as for a better description of phenotypes. Furthermore, since the LOVD databases have a webservice (api) the data can be accessed electronically and used to automatically annotate exome/whole genome sequencing data using tools like EBI's Variant Effect Predictor (WEP).

In this study we added further knowledge and new observations to ADPKD disease by explaining the molecular defect in 352 patients, with the majority of them (208/352) carrying a new pathogenic variant. The large majority of pathogenic variant was constituted by single base substitutions or small in-dels, while large rearrangements displayed a low frequency $(<2 \%)$. Molecular analysis helped us to confirm the diagnosis in clinically uncertain/atypical cases, to exclude the presence of a variant in donors for kidney transplantation and to offer genetic counselling in at risk families. To this respect, by using genetic testing in relatives of probands with a 
A

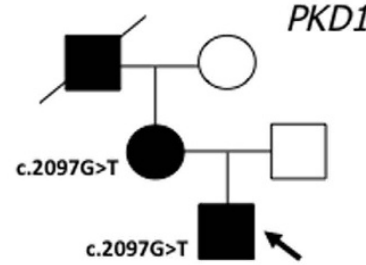

C

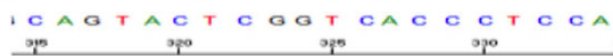

c.2097G

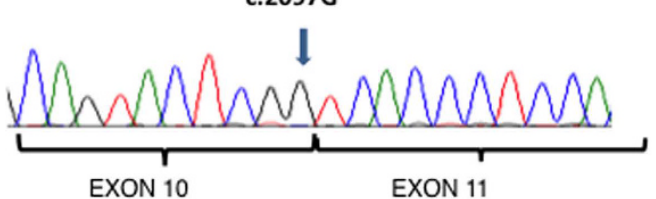

B

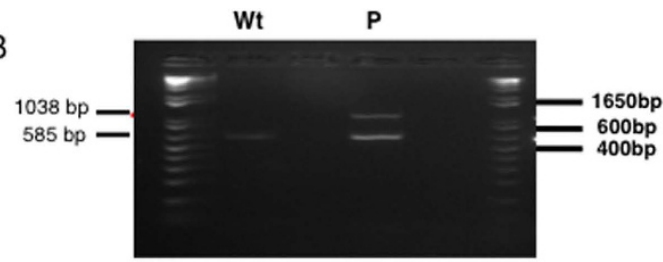

D

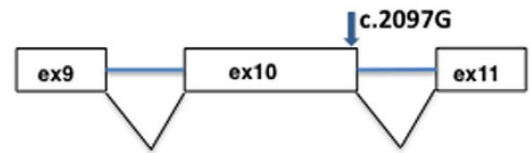

c.2097T

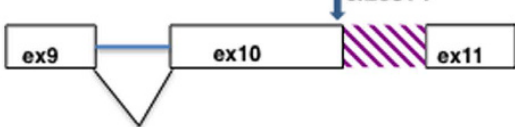

mutant cDNA (1038bp fragment)

oAOTAOTETOTOTOTOOCOCT

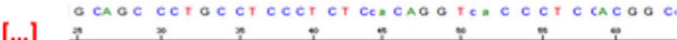

c.2097T

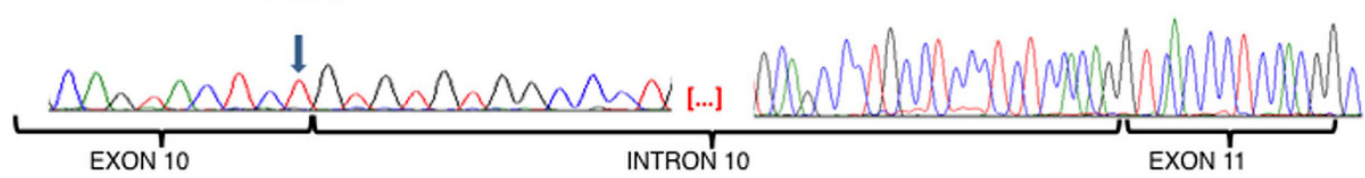

Figure 2. (A) Pedigree. In this family, the proband (arrowed) inherited a synonymous PKD1 variant (c.2097G> T, p. Ser699=) from his mother. (B) RT-PCR from whole blood RNA. In the control sample (Wt), only the expected $585 \mathrm{bp}$ fragment was present. In the patient sample $(\mathrm{P})$, an additional $1038 \mathrm{bp}$ abnormal fragment was amplified. (C) Sequencing of the regular and abnormal fragments from the patient sample. In the upper panel, the sequence of the $585 \mathrm{bp}$ normal c.2097G allele cDNA showing the adjacent exons 10 and 11, correctly spliced; in the lower panel, the sequence of the abnormal $1038 \mathrm{bp}$ cDNA showing that in the mutated allele c.2097T, intron 10 has been completely retained in the transcript. (D) A scheme of the spliced regions in the normal and mutant alleles. Filled symbol: ADPKD; empty symbol: healthy subject.

\begin{tabular}{|l|c|c|c|}
\hline & PKD1 & PKD2 & PKD1 + PKD2 \\
\hline N. of patients with 1 variant & 216 & 70 & \\
\hline N. of patients with 2 variants & 118 & 3 & \\
\hline N. of patients with 3 variants & 39 & 0 & \\
\hline N. of patients with 4 variants & 14 & 0 & \\
\hline N. of patients with variants in both genes & & & 45 \\
\hline
\end{tabular}

Table 6. Patients with multiple novel variants.

pathogenic variant, we were able to prove 97 individuals to be free of the disease and 91 individuals diagnosed with ADPKD.

Genetic analysis revealed an extraordinary high degree of allelic variability, especially in $P K D 1$; we were able to identify a definite pathogenic variant in $54 \%$ of cases. In order to improve counselling to patients and their relatives, we undertook the analysis of the remaining unclassified variants with predictive tools, according to the criteria adopted by Audrezet MP and co-authors ${ }^{9}$. These tools evaluate the effect of variants in light of conservation in a multi-sequence alignment. Data on segregation analysis, co-occurrence with a pathogenic variant, frequency analysis in controls, analysis of transcripts and in-silico evaluation of intronic variants were taken into account to add evidence and were very helpful for counselling. Classification of highly likely or likely pathogenic variants in $26 \%$ of patients raised the total detection level to $80 \%$.

In addition to classified variants, other novel variants have been found, but their classification was uncertain with the criteria adopted, especially for missense changes with a score below the fixed threshold (122 in PKD1 and 7 in PKD2). Thanks to the contextual analysis of $P K D 1$ and $P K D 2$, a number of variants in the group with uncertain significance (Suppl. Table S6), when they were the only one present, might be considered as a strong candidate pathogenic variant, nevertheless we did not consider this observation sufficient to assess pathogenicity. In general, classification of missense variants remains cumbersome because it is not feasible to perform a functional study for each variant, sometimes due to experimental limitations, like for Polycystin-1, but more often due 


\begin{tabular}{|c|c|c|c|c|c|c|}
\hline N. & CDNA & Protein & Trans & Score & $\begin{array}{l}\text { Prediction for novel } \\
\text { variants }\end{array}$ & $\begin{array}{l}\text { Novel/PKDB } \\
\text { classification }\end{array}$ \\
\hline & PKD1 & & & & & \\
\hline \multirow{2}{*}{1} & c.8689_8690insCCAACTCCG & p.Val2897delinsAlaAsnSer & $\mathrm{y}$ & & $\begin{array}{l}\text { highly likely } \\
\text { pathogenic }\end{array}$ & novel \\
\hline & c.12112insG & p.Val4038Glyfs*118 & & & definitely pathogenic & novel \\
\hline \multirow{2}{*}{2} & c.7859_7863delCGAG & p.Asn2620Ilefs*39 & & & definitely pathogenic & novel \\
\hline & c.7863+1_+4delGTGA & & & & definitely pathogenic & novel \\
\hline \multirow{2}{*}{3} & c. $6050 \mathrm{C}>\mathrm{A}$ & P.Ser2017* & & & & definitely pathogenic \\
\hline & c. $12442 \mathrm{G}>\mathrm{A}$ & p.Glu4148Lys & & 17 & likely pathogenic & novel \\
\hline \multirow{2}{*}{4} & c. $9694 \mathrm{~A}>\mathrm{T}$ & p.Lys3232* & & & definitely pathogenic & novel \\
\hline & c. $10651 \mathrm{C}>\mathrm{T}$ & p.Pro3551Ser & & 20 & likely pathogenic & novel \\
\hline \multirow{2}{*}{5} & c. $11537+2 \mathrm{~T}>\mathrm{A}$ & & & & definitely pathogenic & novel \\
\hline & c. $9829 \mathrm{C}>\mathrm{T}$ & p.Arg3277Cys & & & & likely hypomorphic \\
\hline \multirow{2}{*}{6} & c.6005_6043del39 & p.Val2002_Gln2014del & & & definitely pathogenic & novel \\
\hline & c. $4057 \mathrm{G}>\mathrm{A}$ & p.Gly1353Ser & & 19 & likely pathogenic & novel \\
\hline \multirow{2}{*}{7} & c.2098-2_2109del14 & & & & definitely pathogenic & novel \\
\hline & c. $9739 \mathrm{C}>\mathrm{T}$ & p.Arg3247Cys & & 20 & likely pathogenic & novel \\
\hline \multirow{2}{*}{8} & c.7119C $>\mathrm{G}$ & p.Cys2373Trp & & 20 & likely pathogenic & novel \\
\hline & c. $8405 \mathrm{C}>\mathrm{T}$ & p.Pro2802Leu & & & & likely pathogenic \\
\hline \multirow{4}{*}{9} & c. $11017-10 \mathrm{C}>\mathrm{A}$ & & & & & $\begin{array}{l}\text { highly likely } \\
\text { pathogenic }\end{array}$ \\
\hline & c. $8369 \mathrm{C}>\mathrm{T}$ & p.Pro2790Leu & & 16 & likely pathogenic & novel \\
\hline & c. $12454 \mathrm{~A}>\mathrm{G}$ & p.Lys4152Glu & & 17 & likely pathogenic & novel \\
\hline & PKD1 + PKD2 & & & & & \\
\hline \multirow{3}{*}{10} & PKD1 c.8293C $>\mathrm{T}$ & p.Arg2765Cys & & & & likely hypomorphic \\
\hline & PKD1 c.10043G >A & p.Arg3348Gln & & & & likely pathogenic \\
\hline & PKD2 c.1094+1G >A & & & & & definitely pathogenic \\
\hline \multirow{2}{*}{11} & PKD1 c.6583_6589del7 & p.Cys2195Glyfs*15 & & & & definitely pathogenic \\
\hline & PKD2 c.2392C > T & p.Arg798Cys & & & & likely pathogenic \\
\hline
\end{tabular}

Table 7. Cases with more than 1 variant in PKD1, PKD2 and classified as pathogenic.

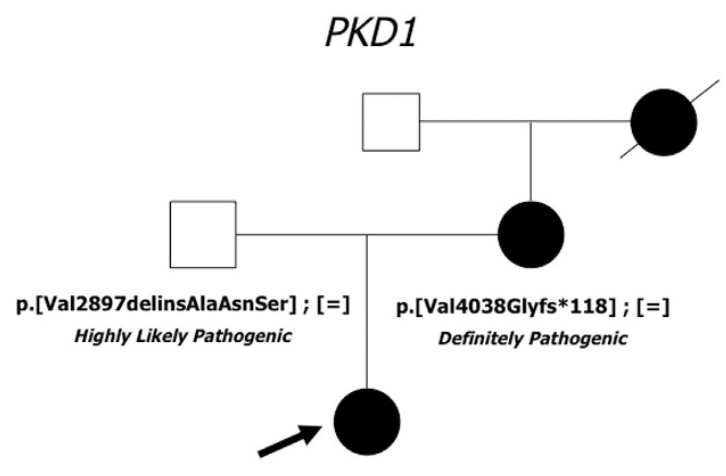

p.[Val2897delinsAlaAsnSer] ; [Val4038Glyfs*118]

Figure 3. In this family, the proband has inherited from the mother a truncating variant, classified as definitely pathogenic, and from the asymptomatic father a del/ins in-frame variant, classified as highly likely pathogenic with the adopted criteria. The proband developed renal insufficiency much earlier than her mother; a RMI renal examination was advised to the father to evaluate a possible subclinical phenotype. Filled symbol: ADPKD; empty symbol: asymptomatic.

to practical and economic barriers. In silico predictors of pathogenicity have been developed and have become popular but they often lack standardization and clinical validation. In the present study we applied classification criteria previously described for $\mathrm{ADPKD}^{9}$ and compared the results to that obtained by Rossetti et al. ${ }^{10}$ for the same missense variants, obtaining a concordance of $83 \%$. This discrepancy may depend not only to in silico prediction algorithm but also to differences in additional evidences contributing to the final scores, since the compared variants were not belonging to the same familiar and clinical contexts.

What we learned from our work is that ADPKD genetics is complicated by the relative high frequency of non-truncating variants, the high allelic heterogeneity with many private variants, the presence of multiple 


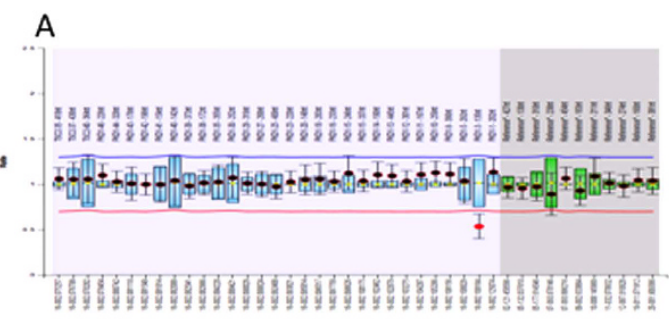

B

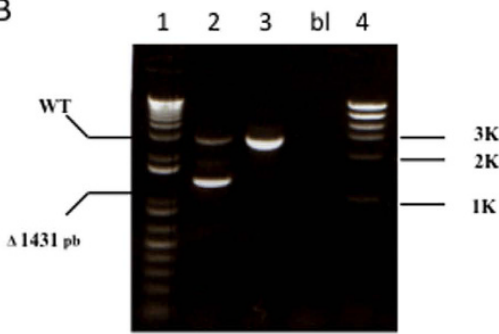

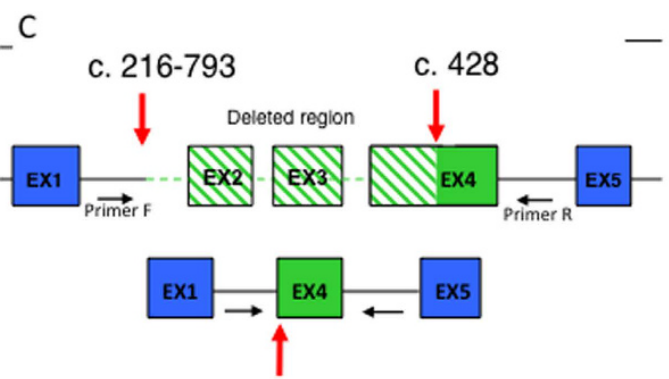

D

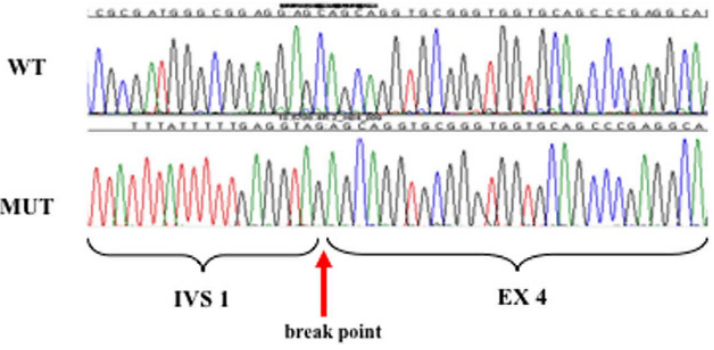

Figure 4. (A) MLPA result suggesting the presence of heterozygous deletion of exon 3 in PKD1; (B) PCR amplification of the deletion borders. In lane 2 the lower fragment is generated by the deleted allele; 2 : patient; 3: healthy control; bl: no DNA; 1, 4: molecular weight markers; (C) schematic representation of the $5^{\prime}$ region of the PKD1 gene in normal (upper) and deleted (lower) alleles. Primer F-2FLR-5'-ATTTTTTGAGAT GGAGCTTCACTCTTGCAGG; primer R-4R 5'-AGCCCTGCCCAGTGTCT. (D) Sequence of the deletion boundaries. Direct sequencing revealed the presence of a $1431 \mathrm{bp}$ deletion, extending from nucleotide c.216793 to c.429 (c.216-793_429del143; http://www.hgvs.org/mutnomen/). The deletion starts 793 bp upstream of exon 2 and stops at position 429 in exon 4, thus causing a frameshift with the production of a truncated putative protein (p.Leu72fs).

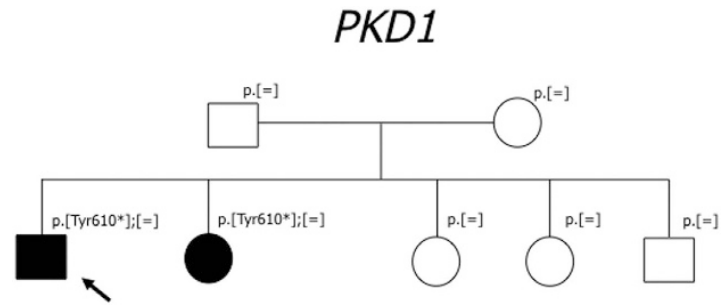

Figure 5. In this family, the proband and his sister are the only ADPKD affected subjects; the proband has kidney and liver multiple cysts, his sister had kidney and liver transplantation at 44 years of age, the parents are healthy and negative to US abdominal examination. Genetic analysis in the family revealed that they did not inherited the truncating variant p.Tyr610*, suggesting the presence of germinal mosaicism in one of the parents. Paternity was confirmed. Filled symbol: ADPKD; empty symbol: healthy.

\begin{tabular}{|l|c|c|c|c|}
\hline \multirow{2}{*}{} & \multicolumn{3}{|c|}{ PKD1 } & PKD2 \\
\cline { 2 - 5 } & $\mathrm{T}^{*}$ & NT $^{\circ}$ & IF $^{*}$ indel $^{\circ}$ & All $^{\circ}$ \\
\hline ESRD n $=44$ & $28(64 \%)$ & $2(4.5 \%)$ & $3(6.8 \%)$ & $3(6.8 \%)$ \\
\hline no ESRD n $=132$ & $62(47 \%)$ & $20(15.2 \%)$ & $3(2.3 \%)$ & $21(16 \%)$ \\
\hline
\end{tabular}

Table 8. Correlation between genotype and ESRD. $*$ Chi-square, $\mathrm{p}$-value $=0.055 .{ }^{\circ}$ Not significant.

variants in the same patient. In this scenario, segregation analysis may be fundamental at least to exclude the pathogenic role of a new variant. Unfortunately, in the present study, contribution of segregation studies for unclassified variants was limited because collection of samples from the families was done more often when a truncating variant was present.

In our opinion, to harmonize and improve assessment of variant pathogenicity for ADPKD it would be important to define a consensus based on standards and possibly on validated criteria. This goal could be supported by improving data-basing and sharing of data and possibly by constituting a multidisciplinary panel of experts, as 


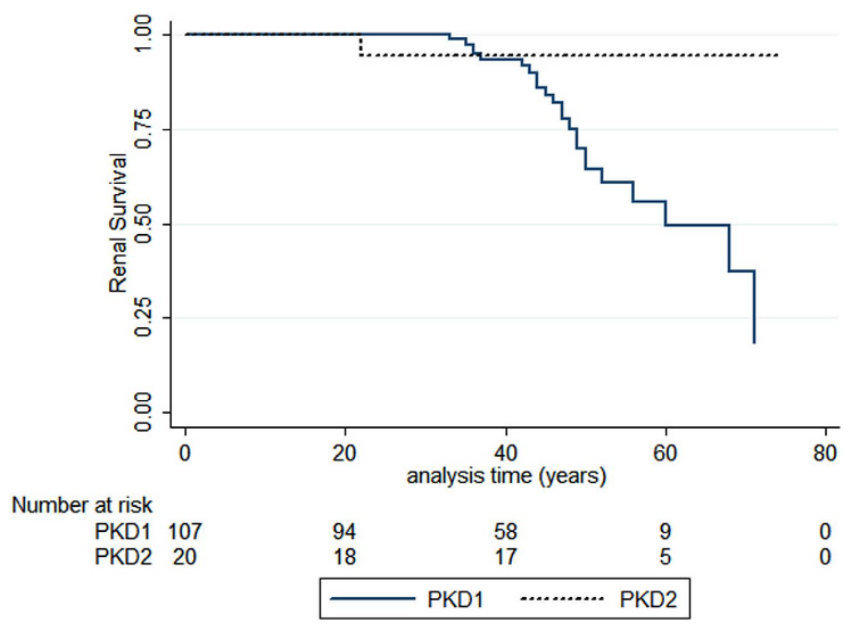

Figure 6. Allelic influence on renal survival. A significant difference in renal survival between patients harboring a $P K D 1$ pathogenic variant and patients with a $P K D 2$ pathogenic variant was observed. Log rank test: Chi-square 5.47, $\mathrm{p}=0.0194$.

already experienced for other human diseases (i.e. InSiGHT's Variant Interpretation Committee) ${ }^{17}$. Databasing is becoming a very important resource for the clinic and for the research; in particular it may be fundamental in the classification and stratification of clinical phenotypes and to not disperse the amount of data that are continuously produced by clinical laboratories.

\section{Patients and Methods}

Patients. The analysis of PKD genes was performed in a cohort of 440 unrelated Italian patients with ADPKD and 203 family relatives, for a total 643 subjects. Among the 440 patients, 320 with a familial positive history, 120 patients with no reported familiarity. Patients were collected in a period of seven years; all the examined subjects gave their written informed consent for genetic testing, data treatment and storage, approved by the Institutional Quality Assessment committee. All the methods were performed in accordance with the guidelines and the experimental protocols approved by the San Raffaele Hospital Istitutional Quality Assessment committee (IQNet IT-2337). In Table 9, phenotypic details on our study patients are reported (age, gender, sCr, eGFR and CDK stages, 1 to 5, at the time of testing). Also available data on extra-renal manifestations associated with ADPKD (hypertension and liver cysts) are reported in Table 9. The patients were referred to the genomic facility from the recruiting groups according to three main modalities and diagnostic questions: i) patients with a family history of ADPKD and positive diagnostic criteria (Research Track); ii) patients with a positive family history at risk for ADPKD but not responding to clinical diagnostic criteria (Diagnostic Track); iii) patients without a family history but a suggestive renal cystic phenotype (Differential Diagnosis Track).

The clinical criteria in the presence of a family history were derived with minor modifications from previous works ${ }^{18,19}$. In brief, in the presence of a positive family history, patients were considered affected if the ultrasound reported 3 cysts or more unilaterally or bilaterally in the age class 15-39 years; more than two cyst per each kidney for subjects in the age class 40-59 years; four or more cysts in each kidney was required for individuals older than $60 \mathrm{yr}^{18}$. For patients analyzed by MRI in the presence of positive family history more than $10 \mathrm{cysts}$ as total number in both kidneys was needed to confirm the diagnosis in patients older than 15 years $^{19}$.

Genetic analysis. Genetic analysis was performed on genomic DNA extracted from peripheral blood on a DNA automatic extractor (Maxwell, Promega, Madison, WI) with the DNA purification kit (Promega), following the manufacturer's instructions.

Sanger direct sequencing. For all the patients, PKD1 and PKD2 (MIM-601313 and 613095) whole coding regions and exon junctions were analysed by directly sequencing PCR products with a Sanger protocol, on both strands. To amplify the functional PKD1, avoiding amplification from pseudo-genes, we used a first round of Long Range PCR using primers and conditions previously described ${ }^{7}$ and specific for the functional PKD1. The 8 Long Range PCR products were then amplified in 43 nested reactions to obtain PCR amplified products corresponding to exons 1-33 and exon-intron junctions. Exons 34 to 46 of PKD1 as well as the 15 exons of PKD2 were amplified from genomic DNA in a single PCR round because these regions are not duplicated in pseudo-genes. PCR conditions and primers are listed in Supplementary Table S9; new PKD1-specific primers for nested PCR have been designed taking into account a multiple alignment (Lasergene) between PKD1 and the 6 pseudogenes.

PCR, nested PCR and sequencing reactions were set up on a liquid handling system (Biomeck FX, Beckman-Coulter) according to protocols developed in the laboratory. PCR and sequencing reactions were purified with Ampure and CleanSeq (Agencourt, Beckman-Coulter) on the FX platform. Dye terminator reaction sequences were loaded on a $3730 \mathrm{AB}$ (Applied Biosystems Inc., Foster City, CA) automatic sequencer. Called sequences were aligned to the reference ENSG00000008710 and ENSG00000118762 with the Sequencer v.5.0 


\begin{tabular}{|c|c|}
\hline Total number of study patients & 643 \\
\hline Total number of study families/probands & 440 \\
\hline Mean age, yr (SD) & $42+/-20$ \\
\hline \multicolumn{2}{|l|}{ Gender } \\
\hline Men & $227(52 \%)$ \\
\hline Women & $213(48 \%)$ \\
\hline \multicolumn{2}{|l|}{ CDK stage $\mathrm{n}=234$} \\
\hline 1 & $80(34 \%)$ \\
\hline 2 & $61(26 \%)$ \\
\hline 3 & $28(12 \%)$ \\
\hline 4 & $9(4 \%)$ \\
\hline 5 & $56(24 \%)$ \\
\hline \multicolumn{2}{|l|}{ Extra-renal manifestations } \\
\hline Hypertension $n=129$ & $75(58 \%)$ \\
\hline Liver cysts $n=133$ & $81(61 \%)$ \\
\hline Families/probands with no variants & $25(6 \%)$ \\
\hline Families/probands with VUS & $63(14 \%)$ \\
\hline Families/probands with pathogenic variants & $352(80 \%)$ \\
\hline Families/probands with PKD2 pathogenic variants & $51(12 \%)$ \\
\hline men & $26(51 \%)$ \\
\hline women & $25(49 \%)$ \\
\hline truncating & $39(11 \%)$ \\
\hline not-truncating & $11(3.1 \%)$ \\
\hline IF indel & $1(0.3 \%)$ \\
\hline Families/probands with PKD1 pathogenic variants & $301(68 \%)$ \\
\hline men & $152(50 \%)$ \\
\hline women & $149(50 \%)$ \\
\hline truncating & $196(55.7 \%)$ \\
\hline not-truncating & $81(23 \%)$ \\
\hline IF indel & $24(6.8 \%)$ \\
\hline
\end{tabular}

Table 9. Characteristics of the study cohort. *eGFR calculated from serum creatinine measurements, milliliters/minute per $1.73 \mathrm{~m}^{2}$.

(Gene Codes Corporation, Ann Arbor, MI). Gene variants were named according to the standard nomenclature (Human Genome Variation Society, www.hgvs.org). The Autosomal Dominant Polycystic Kidney Disease Data Base, public genomic browsers (Ensembl, NCBI, 1000Genomes) and data published in the literature were searched to look for already described variants and for variant classification. All of the sequence changes identified have been confirmed on a second DNA extraction from the same blood sample.

Multiple Ligation Probe Assay. In patients with no variants detected with Sanger sequencing, a MLPA analysis has been performed with the MRC-Holland SALSA MLPA P351 PKD1 and P352 PKD1-PKD2 probe-mix, following the manufacturer's instructions.

Identification of deletion breakpoints was performed by specific amplification of deletion borders and direct sequencing.

RNA analysis. We used Paxgene ${ }^{\mathrm{TM}}$ Blood RNA System (PreAnalytiX, Hombrechtikon, $\mathrm{CH}$ ), which consists of an evacuated Paxgene ${ }^{\mathrm{TM}}$ RNA tube (PAX tube) for blood collection and a processing kit (PAXgene Blood RNA Kit, Qiagen) for isolation of total RNA from whole blood.

$1 \mu \mathrm{g}$ RNA was reverse transcribed into cDNA with random hexamer using MuLV reverse transcriptase (Roche) and amplified by PCR using PKD1 exonic primers: forward $5^{\prime}$-GCGTCTGAGCCGTGAAG $-3^{\prime}$ and reverse $5^{\prime}$-GCCCAGGCAGCCGCAGT $-3^{\prime}$ located in exons 9 and 11 respectively, and yielding an amplicon of $585 \mathrm{bp}$. Direct sequencing of the amplicon was carried out as described above.

Classification of variants. Variants identified in the present study were classified as previously described ${ }^{9}$ : i) large rearrangements, nonsense, frameshift deletions, insertions or indels, variants affecting canonical splice-sites, in-frame changes of $\geq 5$ amino acids were classified as definite pathogenic variants; ii) in-frame changes of $<5$ amino acids, missense and atypical splicing previously reported in patients, segregating with the disease were classified as highly likely pathogenic variants; iii) novel missense variants with a combined score $\geq 14$ and intronic variants at positions +3 and -3 from the exon were classified as likely pathogenic variants; iv) novel missense variants with a combined 


\begin{tabular}{|c|c|c|}
\hline \multicolumn{3}{|l|}{ PUBLIC DATA } \\
\hline GENERAL INFO & PREDICTIONS & KEY CLINICAL DATA \\
\hline Gene name & Grantham & End Stage Renal Disease \\
\hline Reference sequence & A-GDGV & (ESRD) \\
\hline \multirow[t]{2}{*}{ Links } & Polyphen2 & Type of Renal Replace Therapy \\
\hline & SIFT & Renal cysts \\
\hline VARIANT INFO & Mutation Taster & Extra-renal cysts \\
\hline Exon & Effect & Cerebral berry aneurism \\
\hline DNA change (cDNA) & & Hypertension \\
\hline DNA change (genomic) & PATIENT INFO & Macrohematuria \\
\hline Published as & Gender & Nephrolithiasis \\
\hline RNA change & Geographic Origin & Creatinine level (mg/dl) \\
\hline Protein & Ethnic Origin & Ellipsoid US volume \\
\hline DB ID & Consanguinity & Ellipsoid MRI volume \\
\hline Allele transmission & Inheritance & Multi-slice MRI volume \\
\hline Variant remarks & Disease & \\
\hline Pub Med reference & Disease status & \\
\hline Frequency & Age at diagnosis & \\
\hline Re-site & Age at ESRD & NON-PUBLIC DATA \\
\hline Genetic origin & Age of death & Individual ID \\
\hline Segregation & & Family ID \\
\hline dbSNP rs & & Clinic ID \\
\hline Template & & Individual remarks \\
\hline \multicolumn{3}{|l|}{ Technique } \\
\hline Tissue & & \\
\hline
\end{tabular}

Table 10. Database Contents.

score $<14$ were classified as variants of uncertain significance; $v$ ) novel synonymous variants and deep intronic variants were classified as likely non pathogenic; vi) known common or rare variants previously classified as likely neutral were classified as benign variants.

To give a meaning to missense variations, we applied the criteria adopted by Audrezet and co-authors ${ }^{9}$, using different prediction software: the Grantham matrix scoring system Align Grantham Variation Grantham Deviation (A-GVGD) ${ }^{20}$, PolyPhen2 ${ }^{21,22}$, Sorting Intolerant from Tolerant (SIFT) ${ }^{23,24}$, and Mutation Taster ${ }^{25}$. All of them base their prediction on phylogenetic and structural information. They use as input file both the protein itself (PolyPhen2 and SIFT) searching automatically for orthologs and homologs, or a list of orthologous proteins (Homo sapiens, Rattus norvegicus, Gallus gallus, Mus musculus, Takifugu rubripes, Danio rerio) previously chosen (SIFT and AGVGD). To strengthen the results, also the conservation scores PhyloP ${ }^{26}$ and phastCons $^{27,28}$ have been calculated. Moreover, the segregation of the variant in affected members of the family raises the pathogenicity.

For known missense variants, a comparison of the scores obtained and their previous classification from the ADPKD database, (http://pkdb.mayo.edu) ${ }^{4}$, was performed.

Statistical analyses. We examined ESRD by Chi-square testing in subjects with different classes of pathogenic variants in PKD1 and PKD2. We compared the age at ESRD by Kaplan-Meier analysis with Log-rank testing in subjects with pathogenic variants in PKD1 and PKD2.

Databasing. As a database for the collected $P K D 1$ and $P K D 2$ gene variants we selected the internationally linked LOVD version 3 platform. LOVD is structured in agreement with all existing guidelines of the HGVS and $\mathrm{HVP}^{29}$ and allows collection, annotation and classification of all variants detected, as well as storing key clinical data, described based on the Human Phenotype Ontology (HPO) phenotypic descriptors ${ }^{30}$. Use of the HPO descriptors may warrant a higher homogeneity especially when data are shared among a number of clinics. The databases can be accessed using the urls http://www.LOVD.nl/PKD1 and http://www.LOVD.nl/PKD2 and through LOVD's webservice (api). The structure, documentation, and operability of the DB are available on-line.

The complete list of contents is reported in Table 10. Contents refer to variant and patient data and are mostly public with the exception of some individual annotations as well as identification codes. Clinical departments contributed individual and clinical data.

\section{References}

1. Torres, V. E., Harris, P. C. \& Pirson, Y. Autosomal dominant polycystic kidney disease. The Lancet 369, 1287-1301 (2007).

2. Bergmann, C. \& Zerres, K. Early manifestations of polycystic kidney disease. The Lancet 369, 2157 (2007).

3. Bergmann, C., Ortiz Bruchle, N., Frank, V., Rehder, H. \& Zerres, K. Perinatal deaths in a family with autosomal dominant polycystic kidney disease and a PKD2 mutation. N. Engl. J. Med. 359, 318-319 (2008). 
4. Rossetti, S. et al. Incompletely penetrant PKD1 alleles suggest a role for gene dosage in cyst initiation in polycystic kidney disease. Kidney Int. 75, 848-855 (2009).

5. Vujic, M. et al. Incompletely penetrant PKD1 alleles mimic the renal manifestations of ARPKD. J. Am. Soc. Nephrol. 21, 1097-1102 (2010).

6. Cornec-Le Gall, E. et al. Type of PKD1 mutation influences renal outcome in ADPKD. J. Am. Soc. Nephrol. 24, 1006-1013 (2013).

7. Phakdeekitcharoen, B., Watnick, T. J. \& Germino, G. G. Mutation analysis of the entire replicated portion of PKD1 using genomic DNA samples. J. Am. Soc. Nephrol. 12, 955-963 (2001).

8. Rossetti, S. et al. A complete mutation screen of the ADPKD genes by DHPLC. Kidney Int. 61, 1588-1599 (2002).

9. Audrezet, M. P. et al. Autosomal Dominant Polycystic Kidney Disease. Comprehensive Mutation Analysis of PKD1 and PKD2 in 700 Unrelated Patients. Hum. Mutat. 33, 1239-1250 (2012).

10. Rossetti, S. et al. Comprehensive molecular diagnostics in autosomal dominant polycystic kidney diasease. J. Am. Soc. Nephrol. 18, 2143-2160 (2007).

11. Hwang, Y.-H. et al. Refining genotype-phenotype correlation in Autosomal Dominant Polycystic Kidney Disease. J Am Soc Nephrol 27, doi: 10.1681/ASN.2015060648 (2015).

12. Heyer C. M. et al. Predicted mutation strength of nontruncating PKD1 mutations aids genotype-phenotypes correlations in Autoosomal Dominant Polycystic Kidney Disease. J Am Soc Nephrol 27, doi: 10.1681/ASN2015050583 (2016).

13. Velinov, M. et al. Polycystic kidneys and $\operatorname{del}(4)(\mathrm{q} 21.1 \mathrm{q} 21.3)$ : further delineation of a distinct phenotype. Eur. J. Med. Genet. 48, $51-55(2005)$

14. Consugar, M. B. et al. Characterization of large rearrangements in autosomal dominant polycystic kidney disease and the PKD1/ TSC2 contiguous gene syndrome. Kidney Int. 74, 1468-1479 (2008).

15. Ariyurek, Y. et al. Large deletions in the polycystic kidney disease 1 (PKD1) gene. Hum. Mutat. 23, 99 (2004).

16. Fokkema, I. F. et al. LOVD v.2.0: the next generation in gene variant databases. Hum. Mutat. 32, 557-563 (2011).

17. Thompson, B. A. et al. Application of a 5 -tiered scheme for standardized classification of 2360 unique mismatch repair gene variants in the InSiGHT locus-specific database. Nat. Genet. 46, 107-115 (2013).

18. Pei, Y. et al. Unified criteria for ultrasonographic diagnosis of ADPKD. J. Am. Soc. Nephrol. 20, 205-212 (2009).

19. Pei, Y. et al. Imaging-based diagnosis of autosomal dominant polycistic kidney disease. J Am Soc Nephrol 26, 746-53 (2015).

20. Tavtigian, S. V., Greenblatt, M. S., Lesueur, F. \& Byrnes, G. B. In silico analysis of missense substitutions using sequence-alignment based methods. Hum. Mutat. 29, 1327-1336 (2008).

21. Sunyaev, S. R. et al. PSIC: profile extraction from sequence alignments with position-specific counts of independent observations. Protein. Eng. 12, 387-394 (1999).

22. Ramensky, V., Bork, P. \& Sunyaev, S. Human non-synonymous SNPs: server and survey. Nucleic Acids Res. 30, 3894-3900 (2002).

23. Ng, P. C. \& Henikoff, S. Predicting deleterious amino acid substitutions. Genome Res. 11, 863-874 (2001).

24. Ng, P. C. \& Henikoff, S. Accounting for human polymorphisms predicted to affect protein function. Genome Res. 12, 436-46 (2002).

25. Schwarz, J. M., Rodelsperger, C., Schuelke, M. \& Seelow, D. MutationTaster evaluates disease-causing potential of sequence alterations. Nat. Methods 7, 575-576 (2010).

26. Pollard, K. et al. Detection of nonneutral substitution rates on mammalian phylogenies. Genome Res. 20, 110-121 (2010).

27. Siepel, A. \& Haussler, D., Phylogenetic hidden Markov models. In Nielsen, R. ed., Statistical Methods in Molecular Evolution, pp. 325-351, Springer, New York. (2005).

28. Siepel, A. et al. Evolutionarily conserved elements in vertebrate, insect, worm, and yeast genomes. Genome Res. 15, 1034-1050 (2005).

29. Vihinen, M., den Dunnen, J. T., Dalgleish, R. \& Cotton, R. G. Guidelines for establishing locus specific databases. Hum. Mutat. 33, 298-305 (2012)

30. Köhler, S. et al. The Human Phenotype Ontology project: linking molecular biology and disease through phenotype data. Nucleic Acid Research 42, D966-74 (2014).

\section{Acknowledgements}

Thank to the patients and their families and to the collaboration of the Polycystic Kidney Italian Association (AIRP, www.renepolicistico.it). Thank to Marcella Mongiana, Giuseppe Bellantuono, Antonella Fantini and other members of Laboratory of Clinical Molecular Biology for technical contribution. Thank to Elena Brioni of the Nephrology Dept. of Ospedale San Raffaele for her valued activity in the clinics. Partially supported by grants from the Ministry of Health (RF08-LVH2) and Ministry of Instruction, University and Research (MIUR2007FJRB7H).

\section{Author Contributions}

P.C., S.C., F.R., A.B. and M.F. designed, performed and analysed genetic data; J.T.d.D., R.M. and S.S. contributed to the db design and population; R.M., P.M., C.I., A.E., S.N. P.M., F.T., R.C., F.S., E.B. and M.T.S.A. contributed the clinical characterization; P.C. wrote the manuscript; P.C. and S.C. created figures. All authors reviewed the manuscript.

\section{Additional Information}

Supplementary information accompanies this paper at http://www.nature.com/srep

Competing financial interests: The authors declare no competing financial interests.

How to cite this article: Carrera, P. et al. Deciphering Variability of PKD1 and PKD2 in an Italian Cohort of 643 Patients with Autosomal Dominant Polycystic Kidney Disease (ADPKD). Sci. Rep. 6, 30850; doi: 10.1038/srep30850 (2016).

This work is licensed under a Creative Commons Attribution 4.0 International License. The images or other third party material in this article are included in the article's Creative Commons license, unless indicated otherwise in the credit line; if the material is not included under the Creative Commons license, users will need to obtain permission from the license holder to reproduce the material. To view a copy of this license, visit http://creativecommons.org/licenses/by/4.0/

(c) The Author(s) 2016 